\title{
Selection Criteria for Breast Cancer Chemoprevention Subjects
}

\author{
Mack T. Ruffin IV, MD, MPH', David A. August, MD², Gary J. Kelloff, MD³, \\ Charles W. Boone, $\mathrm{MD}^{3}$, Barbara L. Weber, $\mathrm{MD}^{4}$, and Dean E. Brenner, $\mathrm{MD}^{5}$
}

1 Department of Family Practice, University of Michigan Medical School, and Department of Epidemiology, University of Michigan School of Public Health, Ann Arbor, MI 48109-0708

2 Department of Surgery, University of Michigan Medical School, University of Michigan Medical Center, Ann Arbor MI 48109-0331

3 Chemoprevention Investigational Studies Branch, Division of Cancer Prevention and Control, National Cancer Institute, Bethesda, MD 20892

4 Department of Internal Medicine, University of Michigan Medical School, Ann Arbor, MI 48109-0680

5 Departments of Internal Medicine and Pharmacology, University of Michigan Medical School, Medical Service, VA Medical Center, Ann Arbor, MI 48109-0504

\begin{abstract}
Early phase chemoprevention trials differ from standard therapeutic clinical trials because asymptomatic, healthy people are treated with a potentially toxic intervention for a prolonged period of time. Current subject selection protocols have relied upon epidemiological methods to identify highrisk individuals. Most available data provide risk estimates for various individual risk factors, but few have reported risk estimates for combinations of risk factors. Selection criteria for the large tamoxifen intervention trial (NSABP P1) were developed from the work of Gail et al. [1]. The Gail model takes into account non-genetic factors (e.g., nulliparity, age at menarche, preexisting pathological conditions) and genetic factors (family history). Using a lifetime risk of $10 \%$ of developing breast cancer as a standard to intervene, NSABP P1 uses the Gail algorithm to select pre- and postmenopausal women for a primary intervention trial. This approach has been criticized for being insufficiently selective (i.e., all women $\geq 60$ yrs), but appears to be the best available method to select subjects for a chemoprevention trial. Other approaches have been based on identification of very high-risk women with acknowledged pathologic conditions [lobular carcinoma in situ, ductal carcinoma in situ (DCIS)]. Attempting to use these proliferative lesions as pathologic endpoints for drug effect has not been attempted. DCIS as a risk factor for tamoxifen intervention was excluded because of controversies over its management and because of frequent difficulties in distinguishing microinvasive from non-invasive lesions. Women treated for early stage breast cancer (Stage I) may be subjects for early stage chemopreventive interventions.

We propose the use of intermediate endpoint biomarkers and genetic markers as entry criteria for early phase chemoprevention trials. For colorectal cancer chemoprevention, we have used a two-step selection process. The first step was based on epidemiologic risk assessment. Entry into the study required that a potential intermediate biomarker be positive and quantifiable. The relationship between modulation of a pre-transformational biomarker and development of cancer ultimately needs proof in a primary interventional trial; however, this methodology may permit screening of potential
\end{abstract}

Address correspondence to Dean E. Brenner, MD, Director, Cancer Chemoprevention Program, University of Michigan Medical Center, Internal Medicine, Hematology/Oncology Division, UpJohn Center, Room 4713, 1310 East Catherine Drive, Ann Arbor, MI 48109.

(C) 1993 Wiley-Liss, Inc. 
chemopreventive agents at lower cost and more rapid turn-around times. In early chemopreventive agent testing for breast cancer chemoprevention, we propose a similar two-step procedure. Epidemiological and/or pathological criteria for risk would be followed by a procedure to obtain cellular material. The cellular material would be assayed for pre-transformational cellular change.

Identifying predictive genes in familial breast cancer cohorts such as the modified BRCA1 gene promises to select individuals at high familial and potentially physiological or environmental risk. The identification o re abnormal gene product in individuals and families will provide another important group of subjects for chemopreventive interventions. The identification of high-risk subjects for breast cancer chemoprevention, particularly those with familial genetic risk, carries important ethical problems. Such women may have difficulties obtaining health and life insurance, deciding to have children, and obtaining work. Chemoprevention trials with genetic selection criteria will need to develop methods of dealing with these issues. (c) 1993 Wiley-Liss, Inc.

Key words: Breast cancer risk, chemoprevention, intermediate biomarkers

1. Gail MH, Brinton LA, Byar DP, Corle DK, Green SB, Schairer C, Mulvihill JJ. (1989) Projecting individualized probabilities of developing breast cancer for white females who are being examined annually. J Natl Cancer Inst 81:1879-1886.

Chemoprevention trials differ in intent and, therefore, selection criteria for subjects. Some chemoprevention trials are designed to intervene in healthy but high-risk populations. In this group of subjects, toxicity is intolerable and selection criteria revolve around subjects who may be genetically or biochemically at higher risk than others for developing cancer. Other chemoprevention trials are designed to prevent recurrence or new primary tumors in previously treated cancer patients. In the latter group of subjects, some toxicity may be tolerated. In both types of trials, selection criteria must be carefully defined so that the potential benefits of the intervention justify the toxicologic risk while ensuring that there will be a sufficient pool of subjects to achieve the statistical goals of the trial design.

Selection of subjects for breast cancer chemoprevention is further complicated by problems of age (premenopausal versus postmenopausal populations), choice of an appropriate cancer risk level for subjects who may require repeated tissue sampling procedures, exposure to potentially toxic drugs, fertility, and the teratogenic risks in a child-bearing population, as well as the potential genetic and sociological problems with having sufficiently high risk for breast cancer to warrant inclusion in a risk-reduction trial at a young age. Appropriate chemopreventive doses for potential pharmacologic interventions will be identified by assessing biochemical or biological endpoints in tissue specimens. These Phase I and II trials will require populations of adherent subjects for whom the risk is physically, psychologically, and ethically acceptable.

Usual endpoints for cancer clinical trials are reduction in tumor size or statistically increased survival in populations whose survival is limited. Such trials may be completed quickly with measurable surrogate (e.g., response of tumor mass) or primary endpoints (e.g., survival after a new treatment compared with survival after a control treatment). Cancer chemoprevention trials pose unique problems in clinical trial design. For chemopreventive interventions, no such easily measured endpoints exist. We must rely on events that happen years later, such as the development of cancer. Complex biostatistical and epidemiological tools are necessary in massive study populations in order to prove clinical efficacy. Such large trials are expensive and impractical in the process of screening many new, promising approaches to chemoprevention. In order to simplify and make chemoprevention trials cost-effective, the identification of intermediate biomarkers as surrogate endpoints for cancer are necessary. Intermediate endpoints will play a key role in subject selection criteria for future chemoprevention trials.

To solve the problem of risk identification for healthy subjects, many investigators have drawn on epidemiologic data that define the risk of developing cancer. Quantitative formulas may be developed to define epidemiologic and genetic risk. Most chemoprevention trials rely upon some epidemiologic risk factors as the basis for 
entry into the study. For early stage chemoprevention trials, the addition of intermediate endpoints as selection criteria is being tested. Finally, genetic risk based on the presence of a known genetic marker may be used in the future to identify high-risk individuals and their families for chemopreventive intervention. We will describe approaches to selecting subjects for chemoprevention trials drawing on our experience, and that of others involved in ongoing chemoprevention trials for high-risk individuals who have not yet developed a primary tumor.

\section{SUBJECTS WITH PRIOR BREAST CANCER}

Patients with locally controlled, low stage (Stage I) breast cancer are an attractive pool of potential subjects for chemoprevention trials. The Milan group [1] has chosen women 1-3 years after resection of Stage I (node-negative) breast cancer as subjects for Phase I and II chemoprevention trials with 4-(hydroxyphenyl)retinamide (fenretinide, 4-HPR). Such patients have an overall relapse-free survival rate of $70-75 \%$ at 10 years [2,3]; however, most recurrences are within 3 years of initial diagnosis and treatment [4]. Chemopreventive interventions in patients with $\geq 25 \%$ risk of recurrence may not be feasible because precancerous lesions in these patients may be too advanced. Selection of women with low stage breast cancer for chemopreventive intervention may be possible using various morphologic and biologic indicators of prognosis to identify women with a low $(<15 \%)$ risk of recurrence [5]. Such criteria include patients with tumors of $<1 \mathrm{~cm}$ in size, positive steroid receptors, and a low proliferative rate as characterized by flow cytometry. The ultimate goal of chemopreventive intervention in this group of women would be the prevention of second primary lesions in either the treated breast or the contralateral breast (estimated risk of new primary distal from the first primary or in the contralateral breast of $1 \%$ per year at 5 years) [6]. Such women might have intermediate biomarker changes consistent with a field defect and could be candidates for early intervention trials.

\section{BENIGN BREAST DISEASE}

Benign breast disease consists of a heterogeneous group of pathologic changes associated with various degrees of breast cancer risk. Breast cancer risk is related to proliferative and nonproliferative conditions [7]. Nonproliferative breast disease (e.g., adenosis, fibroadenoma, fibrosis, mastitis, mild hyperplasia) is less associated with excess risk of breast cancer, whereas the relative risk of proliferative breast disease with or without atypia, is 4.4 [7]. Patients with proliferative breast disease and a family history of breast cancer have a $20 \%$ risk of breast cancer over 15 years [8] and may benefit from chemopreventive interventions.

Ductal carcinoma in situ (DCIS), a proliferation of premalignant epithelial cells confined to the mammary ducts, is regarded as a direct precursor of cancer and is usually treated surgically. The risk of developing invasive breast cancer in women who had breast conservation treatment for DCIS is $7-10 \%$ over 10 years [10]. The concept of multicentricity has been recently questioned [9]. Evidence of unicentricity is less supportive of a cancer "field effect." Since DCIS represents breast cancer at its earliest stage, these women are excellent candidates for chemopreventive interventions using biomarker analysis to estimate the potential for recurrence or primary breast cancer.

A significant body of data supports the hypothesis that LCIS is a marker for increased cancer risk in either breast [11,12]. This group of patients may also be excellent for all phases of chemoprevention trials.

\section{SELECTION OF SUBJECTS WITHOUT PRIOR BREAST CANCER}

\section{Epidemiologic Selection Criteria}

Data from studies of millions of women developing breast cancer provide some insight into the risk of developing breast cancer. Numerous studies have consistently found gender, age, family history, presence of histologic precursor lesions, early age at menarche, late age at menopause, low parity, older age at first birth, history of a previous breast cancer, and radiation exposure to be factors influencing an individual's risk of breast cancer [13-19]. Risk factors can be present separately or in an infinite number of combinations. The direct approach to estimating the absolute probability of developing breast cancer for each risk factor separately or in combination 
is not feasible. Given the available data, the best method is to use epidemiologic models.

\section{Epidemiologic Models for Selection Criteria in Chemoprevention Trials}

Several attempts have been made to devise population-based selection strategies for breast cancer screening [20-26] or estimates of absolute breast cancer risk $[17,27,28]$. The populationbased selection strategies have some common weaknesses. For example, Soini and colleagues [24] constructed a multivariate discriminant which correctly identified $85 \%$ of cases as being high risk, but also included the majority $(65 \%)$ of healthy controls in this high-risk classification. Alexander and colleagues [21] used four risk factors to define a high-risk subgroup of $48 \%$ of women, but it identified only $56 \%$ of all the breast cancers. For cancer screening, most would agree that the sensitivity of any selection protocol should not fall below $80-90 \%$, otherwise the intent of screening would be nullified. The same requirements can be used for selection criteria for chemoprevention trials. Studies by Ottman and Anderson $[27,28]$ focused on relatives of women with known breast cancer. This unique population does not consider the vast number of women without family history of breast cancer or other risk factors.

Gail and colleagues [17] developed a model based on 5,998 participants in the Breast Cancer Detection Demonstration Project (BCDDP), to estimate the long-term probability that a woman with a specified combination of risk factors would develop breast cancer. The risk factors identified were age, age at menarche, age at first live birth, the number of previous breast biopsies, and the number of first-degree relatives with breast cancer. This model is being used as the selection criteria for the National Surgical Adjuvant Breast and Bowel Project (NSABP P1) trial, setting the minimum relative risk for entry as $10 \%$ lifetime risk. Some potential limitations of this model are the possible overestimation of relative risk due to self-selection of women into the BCDDP program, and the total focus on Caucasian women. The M.D. Anderson group has recently tested the Gail model using SEER data and found it to be predictive of breast cancer risk [29]. This model represents the best one available combining genetic and biological risk factors.

\section{Intermediate Biomarkers in Chemoprevention Trials}

Regardless of the approach used to identify high-risk women for breast cancer chemoprevention studies, one is left with the difficult task of using endpoints such as diagnosis of invasive breast cancer or death from breast cancer. This creates logistical problems with respect to the research design (e.g., 16,000 women in the NSABP P1), many years of intervention and follow-up, and massive resources.

Since cancer is an uncommon event and the duration of time until sufficient subjects develop cancer is long, it is not feasible to perform such an observational trial for the many potential chemopreventive compounds becoming available for clinical trial. Modulation of markers of premalignant cells from individuals at risk reduces the size of population and the time required for study.

The optimal intermediate biological endpoint for an at-risk population will be readily expressed in tissues that are accessible to biopsy or in the plasma, related in some way to the processes of neoplastic transformation and progression, easily measured from small quantities of tissue, quantifiable as a continuous variable, and expected to be modulated by chemopreventive intervention.

To determine chemopreventive efficacy, we propose the use of drug effect, biological, and pathological endpoints. We define a drug effect endpoint as one in which a pharmacologic agent modulates a cell's normal biochemical function. For example, in determining aspirin's chemopreventive effects on colorectal mucosa, we first need to determine whether sufficient drug has reached the appropriate cells to modulate cellular function. Assessment of cyclooxygenase activity through the amount of prostaglandin product in gut tissue constitutes a drug effect endpoint, because it measures a known biochemical target of drug action. Measuring drug effect tells us that the dose and schedule of a potential chemopreventive agent is delivered in sufficient amounts to the target site to cause an effect. This concept is useful in Phase I and II chemoprevention trials. 
We define a biological endpoint as one in which an intervention changes a cellular product which has been identified as a marker for cancer risk. For example, a change in the expression of the oncogene product $\mathrm{p} 21$ is such an endpoint.

We define a pathological endpoint as one in which a defined premalignant lesion, such as cervical intraepithelial neoplasia, measured visually using colposcopy and pathologically by biopsy, reverses after a defined treatment period. Such endpoints are directly on the causal pathway to cancer. As such, pathological endpoints may be used to measure the effects of chemopreventive agents [30, these proceedings] and identify cohorts for chemoprevention trials.

\section{Combined Epidemiologic and Intermediate Biomarker Subject Selection Criteria}

Phase II chemoprevention trials are designed to determine if a potential chemopreventive agent will modulate a biological endpoint as a surrogate for chemopreventive effect. Phase IIa trials identify optimal doses of a putative chemopreventive agent that modulate biological intermediate endpoints through a dose escalation schema, while Phase IIb trials identify the activity of a given chemopreventive agent through modulation of an intermediate endpoint in a randomized trial.

Because the goal of Phase II chemoprevention trials is modulating an intermediate biomarker, we have required a two-step subject selection process. The first step requires sufficiently high epidemiologic risk to provide motivated subjects. The second step requires an intermediate endpoint assay of a biological specimen. Results of the assay should be in the "high risk" range as determined by prior evaluation of the test. By "frontloading" our Phase II trials with a population of subjects who have known changes in a surrogate endpoint, the potential efficacy of a chemopreventive drug may be rapidly tested in a human population.

Such a two-step approach reduces the power requirements necessary to demonstrate a biological change in a cohort of subjects in whom the marker is already theoretically outside the normal population distribution. By attempting to control for large biological variability and using a paired study design wherein each subject serves as her own control, a reduction in the study sample size simplifies the two-step design approach.

The two-step design adds complexity to the on-study stage of a chemoprevention trial-each potential experimental subject must provide a specimen for assay prior to study entry. This process increases the time required for study entry, and reduces the overall efficiency of study execution. Not all subjects who are epidemiologically suitable will have an appropriately altered biological intermediate endpoint for study entry. More samples will need to be processed than the numbers of subjects actually entering the trial. The ratio of specimens versus subjects entering the trial will depend on the rigidity of the epidemiological selection criteria and the assay entry requirements. Cost savings are realized by the smaller numbers of subjects needed to meet power requirements.

\section{Genetic Selection Criteria}

While the contribution of family history to the risk of developing breast cancer has been carefully studied epidemiologically $[15,17,18,22,23]$, the recent work of King et al. [31,32] identified chromosome 17q12-21 as the location of a gene alteration responsible for the development of breast cancer and ovarian cancer by genetic linkage analysis. This gene, which has not been cloned and sequenced to date, is now termed BRCA1. Families with breast and ovarian carcinoma that are thought to have resulted from BRCA1 mutations have been identified by many groups, and some investigators have begun to provide families with information on mutation carrier status [33]. Female carriers of altered BRCA1 are estimated to have an $85 \%$ lifetime risk of developing breast or ovarian cancer or both. More than $50 \%$ of the breast cancers occur prior to age 50 . Inheritance follows classical Mendelian autosomal dominant inheritance patterns $[34,35]$. Inherited BRCA1 germline mutations may account for $2-10 \%$ of all breast cancer. Somatically acquired mutations of BRCA1 or other key loci on chromosome 17 may account for some sporadic cases of breast cancer [36].

The Li-Fraumeni syndrome is a rare familial cancer syndrome resulting from an inherited mutant p53 allele. Inherited mutations in p53 may account for about $1 \%$ of breast cancer 
among patients who are diagnosed before age 40 [37]. An altered estrogen receptor (ER) gene may be responsible for inherited breast cancer. One description of a family with postmenopausal breast cancer linked to ER has been published [38].

When the BRCA1 sequence is known, it will be possible to identify women who carry the high-risk allele. Such women would become candidates for chemopreventive intervention. Currently, women with linkage studies and family histories that support altered BRCA1 inheritance have undergone bilateral mastectomies and oophorectomies in the hope of preventing cancers at these sites. Once the function and product of this gene is determined, gene therapy, as well as chemopreventive intervention, may be a possible treatment for these women.

Of perhaps greater importance is the description of a gene in which acquired somatic mutations may lead to early risk of developing spontaneous, noninherited breast cancer. Since $90 \%$ of breast cancer cases are spontaneous, the development of pre-transformational genetic markers such as altered BRCA1 may be used in future chemoprevention trials as selection criteria. Optimally, genetic mutations used for subject selection for chemoprevention trials should be sufficiently early in carcinogenesis that cells are morphologically normal yet genetically abnormal. Spontaneous BRCA1 mutations, or other potential genetic markers (e.g., ER), have the potential for being key selection criteria for chemoprevention subjects, as well as identifying pharmacologic or genetic materials that might be used to reverse the transformation process.

\section{ETHICAL CONSIDERATIONS}

\section{Psychosocial and Economic Issues}

The psychological, social, reproductive, and economic consequences of having sufficient risk to be selected into chemoprevention trials need careful attention and planning. For example, what are the reproductive consequences in premenopausal women who desire children and carry the high-risk BRCA1 allele? Will such a woman be able to obtain health insurance in our current system given her genetic "diagnosis"? How and when should such information be disclosed to the patient? To her employers? To her family? Will participation in a chemoprevention trial impair a women's ability to obtain employment? Will affected women have difficulties establishing strong, lasting emotional and sexual relationships?

As molecular epidemiology becomes the basis for chemoprevention trial subject selection, we must deal with the ethical consequences of our selection process. The Breast Cancer Center at the University of Michigan has developed a protocol to address the many issues that arise with molecular diagnosis [39]. First, precounseling education and assessment occurs several weeks prior to the planned clinic visit. At this session, a genetic counselor and oncology nurse obtain informed consent and draw blood samples for genetic testing. Risks and benefits of learning molecular risk status are presented in order to permit subjects to make informed decisions about receiving test results. Risks including jeopardizing insurance coverage, the possibility of expensive surgery, disclosure to employers or potential employers, and confidentiality among other family members are thoroughly discussed. Second, results are disclosed by a multidisciplinary team including geneticists, oncologists, genetic counselors, and oncology nurses. The team educates subjects (both gene carriers and noncarriers) about the methods used to detect a genetic defect, explains the test results, discusses future risks for breast cancer, explains medical interventions, chemoprevention or screening options, and provides psychological support options. Third, members of the breast care team and other mental health professionals provide psychological support. There is ample opportunity to discuss emotional responses, and follow-up counseling is provided. Finally, social issues are addressed. The University of Michigan Group has opted not to reveal results to individuals younger than 18 years of age because there are no known medical benefits to a minor knowing that he or she is a gene carrier. It is difficult to determine the emotional maturity required to receive such results. There are no nationally established guidelines for screening minors for late-onset disorders. The potential risks of loss of insurance are discussed with each family at length. A separate shadow file for each patient is established so that the results of genetic testing are not reflected in the hospital chart. 


\section{Chemopreventives as Potential Teratogens in Women of Reproductive Age}

Pharmacologic chemopreventives may have known or unknown teratogenic potential. The problem is especially important when chemopreventive interventions are contemplated in women of reproductive age. We wish to select women with high-risk breast lesions or who carry gene muta is associated with inherited breast cancer for chemoprevention trials. We have supported entry of premenopausal women into chemoprevention trials using compounds that are potential teratogens (e.g., retinoids) for the following reasons: (1) Early stage or high-risk breast lesions are usually discovered in women of reproductive age. We are concerned that, by studying only postmenopausal women, we will select a subset of women who have a different level of risk, and perhaps, different mechanisms of pre-transformational change from premenopausal women. (2) Many Phase II chemoprevention trials are 12 months or less in duration. We feel it is reasonable to demand hormonal methods of birth control for the period of study in premenopausal women. The potential effects of hormonal birth control on breast cellular precancer events should be controlled for in a trial design in which the placebo arm consists of women on hormonal birth control and a placebo chemopreventive. While we would prefer to include a barrier method of birth control in our design, we feel that inclusion of such methods will require additional stratification and increase the complexity and costs of chemoprevention trials. The loss of potential subject accruals due to rejection of hormonal birth control methods would, in our estimation, be minimal.

\section{SUMMARY}

Selection of subjects for interventional chemoprevention trials is currently based on epidemiological criteria. We have proposed a two-step entry procedure that includes an epidemiologic risk assessment followed by demonstration of an intermediate biomarker that may be used as a surrogate endpoint in chemoprevention trials. The development of reproducible molecular diagnostic methods will revolutionize entry criteria for chemoprevention trials by identifying young, high-risk subjects. Identification of abnor- mal genetic products in women with familial breast cancer is an example. Such information may be used to detect precancerous cellular changes in women who will develop spontaneous breast cancer, thus providing more precise selection criteria for chemopreventive interventions.

In a time of turmoil in our medical care system and a new emphasis on preventing deadly diseases such as breast cancer, we must ensure that our subjects do not suffer unnecessarily from the psychological, social, and economic consequences of their genetic inheritance.

\section{ACKNOWLEDGEMENTS}

This work was supported by $\mathrm{NCI}$ Contract CN-15336, NIH MO-1 RR 00042 American Cancer Society Institutional Grant, and the University of Michigan Cancer Center.

\section{REFERENCES}

1. Costa $A$, Malone $W$, Perloff $M$, Buranelli $F$, Campa T, Dossena G, Magni A, Pizzichetta M, Andreoli C, del Vecchio M, Formelli F, Barbieri A. (1989) Tolerability of the synthetic retinoid fenretinide (HPR). Eur J Cancer Clin Oncol 25:805-808.

2. Valagussa P, Bonadonna G, Veronesi U. (1978) Patterns of relapse and survival following radical mastectomy: Analysis of 716 consecutive patients. Cancer 41:1170-1178.

3. Fisher B, Slac N, Katrych D, Wolmark N. (1975) Ten year follow-up results of patients with carcinoma of the breast in a co-operative clinical trial evaluating surgical adjuvant chemotherapy. Surg Gynecol Obstet 140:528-534.

4. Henderson IC, Hayes DF, Parker LM. (1990) Adjuvant systemic therapy for patients with node-negative tumors. Cancer 65:2132-2147.

5. McGuire WL, Clark GM. (1992) Prognostic factors and treatment decisions in axillary-node-negative breast cancer. N Engl J Med 326:1756-1761.

6. Recht A, Silen W, Schnitt S, et al. (1988) Time course of local recurrence following conservative surgery and radiotherapy for early stage breast cancer. Int J Radiat Oncol Bio Phys 15:255-261.

7. Dupont W, Page D. (1985) Risk factors for breast cancer in women with proliferative breast disease. $\mathrm{N}$ Engl J Med 321:146-151.

8. Dupont W, Page D. (1989) Relative risk of breast cancer varies with time since diagnosis of atypical hyperplasia. Hum Pathol 20:725-725.

9. Holland R, Connolly JL, Gelman R, Mravunac M, Hendriks JHCL, Verbeek ALM, Schnitt SJ, Silver B, Boyages J, Harris JR. (1990) The presence of an exten- 
sive intraductal component following a limited excision correlates with prominent residual disease in the remainder of the breast. J Clin Oncol 8:113-118.

10. Van Dongen J, Holland R, Peterse J. (1992) Ductal carinoma in situ of the breast: Second EORTC consensus meeting. Eur I Cancer 28:626-629.

11. Wheeler J, Enterline H, Roseman J. (1974) Lobular carcinoma in situ of the breast. Long-term follow-up. Cancer 34:554-563.

12. Frykberg E, Santiago F, Betsill W, O'Brien P. (1987) Lobular carcinoma in situ of the breast. Surg Gynecol Obstet 164:285-301.

13. Reuter KL, Baker SP, Krolikowski FJ. (1992) Risk factors for breast cancer in women undergoing mammography. AJR 158:273-278.

14. Ingram D, Nottage E, Ng S, Sparrow L, Roberts A, Willcox D. (1989) Obesity and breast disease. The role of the female sex hormones. Cancer 64:1049 1053.

15. Colditz GA. (1993) Epidemiology of breast cancer. Findings from the Nurses' Health Study. Cancer 71: 1480-1489.

16. Layde PM, Webster LA, Baughman AL, Wingo PA, Rubin GL, Ory HW. (1989) The independent associations of parity, age at first full term pregnancy, and duration of breast feeding with the risk of breast cancer. Cancer and Steroid Hormone Study Group. $\mathrm{J}$ Clin Epidemiol 42:963-973.

17. Gail MH, Brinton LA, Byar DP, Corle DK, Green SB, Schairer C, Mulvihill JJ. (1989) Projecting individualized probabilities of developing breast cancer for white females who are being examined annually. J Natl Cancer Inst 81:1879-1886.

18. Sattin RW, Rubin GL, Webster LA. (1985) Family history and the risk of breast cancer. JAMA 253: 1908-1913.

19. Seidman H, Stellman SD, Mushinski MH. (1982) A different perspective on breast cancer risk factors: Some implications of the nonattributable risk. CA 32:301-313.

20. Shapiro S, Goldberg J, Venet L, Strax P. (1973) Risk factors in breast cancer-a prospective study. In Doll P, Vodopija I (eds): "Host Environment Interactions in the Etiology of Cancer in Man." Lyon: IARC Scientific Publications no. 7, pp 169-182.

21. Alexander FE, Roberts MM, Huggins A. (1987) Risk factors for breast cancer with applications for selection for prevalence screen. J Epidemiol Community Health 41:101-106.

22. Schechter MT, Miller AB, Baines CJ, Howe GR. (1986) Selection of women at high risk of breast cancer for initial screening. J Chronic Dis 39:253-260.

23. Farewell VT. (1977) The combined effect of breast cancer risk factors. Cancer 40:931-936.

24. Soini I, Hakama M. (1978) Failure of selective screening for breast cancer by combing risk factors. Int J Cancer 22:275 281 .
25. de Waard F, Rombach JJ, Collette HJA. (1978) The DOM-Project for the early diagnosis of breast cancer in the city of Utrecht, The Netherlands. In Miller AB (ed): "Screening for Cancer." Geneva: IUCC Technical Report Series, vol 40, pp 183-200.

26. Toti A, Piffanelli A, Pacanelli T. (1980) Possible indication of breast cancer risk through discriminant function. Cancer 46:1280-1285.

27. Ottman R, King MC, Pike MC, Henderson BE (1983) Practical guide for estimating risk for familial breast cancer. Lancet 2:556-558.

28. Anderson DE, Badzioch MD. (1985) Risk of familial breast cancer. Cancer 56:383-387.

29. Vogel V, Bondy M, Halabi S, Lustbader E, Lord J, Winn R. (1993) Validation of Gail's Breast Cancer Rist Model. Proc Am Soc Clin Oncol 12:167 (abstract).

30. Boone CW, Kelloff GJ (1993) Intraepithelial neoplasia, surrogate endpoint biomarkers, and cancer prevention. J Cell Biochem 17 (Suppl. F):37-48.

31. Hall JM, Lee MK, Newman B, Morrow JE, Anderson LA, Huey B, King MC (1990) Linkage of early onset breast cancer to chromosome $17 \mathrm{q} 21$. Science 250 : 1684-1689.

32. Narod SA, Feunteun J, Lynch HT, Watson P, Conway T, Lynch J, Lenoir GM (1991) Familial breastovarian cancer locus on chromosome 17q12-23. Lancet 338:82-83.

33. Lynch HT, Watson P. (1992) Genetic counselling and hereditary breast/ovarian cancer. Lancet 339:1181.

34. Smith SA, Easton DF, Ford D, Peto J, Anderson K, Averill D, Stratton M, Ponder M, Pye C, Ponder BA (1993) Genetic heterogeneity and localization of a familial breast-ovarian cancer gene on chromosome 17q12-q21. Am J Hum Genet 52:767-76.

35. Easton DF, Bishop DT, Ford D, Crockford GP. (1993) Genetic linkage analysis in familial breast and ovarian cancer: Results from 214 families. The Breast Cancer Linkage Consortium. Am J Hum Genet 52:678701.

36. Sato T, Akiyama F, Sakamoto G, Kasumi F, Nakamura $Y$ (1991) Accumulation of genetic alterations and progression of primary breast cancer. Cancer Res 51:5794-5799.

37. Malkin D, Li FP, Strong LC, Fraumeni JF Jr, Nelson CE, Kim DH, Kassel J, Gryka MA, Bischoff FZ, Tainsky MA, et al (1990) Germ line p53 mutations in a familial syndrome of breast cancer, sarcomas, and other neoplasms. Science 250:1233-1238.

38. Zuppan PJ, Hall JM, Lee MK, Ponglikitmongkol M, King M-C. (1991) Possible linkage of the estrogen receptor gene breast cancer in a family with lateonset disease. Am J Hum Genet 48:1065-1068.

39. Biesecker BB, Boehnke M, Calzone K, Markel DS, Garber JE, Collins FS, Weber BL. (1993) Genetic counseling for families with inherited susceptibility to breast and ovarian cancer. JAMA 269:1970-1974. 